\title{
The effect of hydrothermal grown zinc oxide nanoparticles as seeds on the properties of nanoripples in zinc oxide thin films
}

\author{
M Seabi ${ }^{1, a,{ }^{*}, \text { T Muller }}{ }^{1, b}$, S Bolokang ${ }^{2,1, c}$, F Cummings, ${ }^{3, \text { d }}$ C Arendse ${ }^{1, e}$ \\ ' Department of Physics and Astronomy, University of the Western Cape, Robert Sobukwe Road, Bellville 7530, South Africa \\ ${ }^{2}$ Advanced Casting Technologies, Light Metals, Materials Science \& Manufacturing, Council for Scientific and Industrial Research, \\ Meiring Naudé Road, Brummeria, Pretoria 0185, South Africa \\ ${ }^{3}$ Electron Microscope Unit, University of the Western Cape, Robert Sobukwe Road, Bellville 7530, South Africa

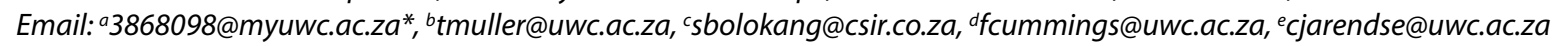

\begin{abstract}
This work reports on a simple approach to improving the optoelectronic properties of Wurtzite $\mathrm{ZnO}$ nanoripples by means of incorporating hydrothermally synthesised $\mathrm{ZnO}$ nanoparticles under controlled synthesis temperature. Initially, $\mathrm{ZnO}$ nanoparticles were investigated and subsequently utilised as seeds to induce ripple growth in spin-coated $\mathrm{ZnO}$ thin films. TEM images illustrated the development of nanospheres at $140^{\circ} \mathrm{C}$. The yield of $\mathrm{ZnO}$ NPs at $180^{\circ} \mathrm{C}$ increased and consisted of a combination of nanorods and nanospheres. Morphologically, seedless ZnO nanoripples showed rugged ends of the nanoripple structures. The SEM images illustrated that the layers uniformly formed on the substrates, and seeding the $\mathrm{ZnO}$ nanoripples caused the nanoripples to elongate. The thickness of the nanoripples thin films showed a decrease with the incorporation of hydrothermally synthesised $\mathrm{ZnO}$ seeds from $134 \mathrm{~nm}$ for unseeded $\mathrm{ZnO}$ nanoripples to $96 \mathrm{~nm}$ at $180^{\circ} \mathrm{C}$. The incorporation of $\mathrm{ZnO}$ NPs seeding treatment increased the transmission of $\mathrm{ZnO}$ nanoripples from $82 \%$ to $92 \%$, leading to untreated $\mathrm{ZnO}$ nanoripples exhibiting a direct band gap of $3.19 \mathrm{eV}$ that increased after seeding to $3.36 \mathrm{eV}$. The change in the band gap to a higher value(s) and increased transparency confirms the progressive improvement of the thin films due to incorporating $\mathrm{ZnO}$ seeding for optoelectronic and photovoltaic applications.
\end{abstract}

Keywords: Hydrothermal Method, Nanoparticles, Nanoripples, Thin Films, Zinc oxide

\section{Introduction}

Semiconductors whose dimensions are nanometre size play a significant role in electronic, photovoltaic, biological sensing, and optical device applications. This is due to their electrical, optical, and chemical properties, which can be tuned by changing the size of the particles. ${ }^{1}$ Zinc oxide $(\mathrm{ZnO})$ is an n-type semiconductor with a direct band gap; it comprises a considerable fraction of ionic bonding. ${ }^{2,3} \mathrm{ZnO}$ has attracted the research field due to its typical and novel properties, such as a wide band gap of $3.37 \mathrm{eV}$ and exciton energy of $60 \mathrm{meV} .{ }^{4}$ Owing to its ability to be effectively used in solid-state light sources and detectors in the blue and UV region of the electromagnetic spectrum, $\mathrm{ZnO}$ occupies a unique position among wide energy band gap semiconductors such as gallium nitride and zinc sulphide. ${ }^{2} \mathrm{ZnO}$ is applied in different fields of science and technology. ${ }^{4}$ Areas such as light-emitting diodes, UV detectors, high sensitive gas sensors, ${ }^{5}$ solar cells, ${ }^{6}$ laser diodes, and photoluminescent devices. ${ }^{4,7}$ Recently, various methods have produced $\mathrm{ZnO}$ powders and thin film structures of controlled morphology and sizes. ${ }^{4}$ Current techniques for synthesising $\mathrm{ZnO}$ nanostructures include chemical vapour deposition (CVD), electrophysical deposition, hydrothermal synthesis, electrochemical synthesis, solution combustion, sol-gel technique, and co-precipitation method. ${ }^{4}$ Hydrothermal synthesis is the promising alternative synthetic method for nano-sized semiconductors due to its low process temperature and its easy route to control particle size. ${ }^{7}$
Moreover, hydrothermal synthesis uses simple equipment, catalystabsence growth, low-cost and environmental friendliness. ${ }^{7}$ The hydrothermal method is applied in microelectronics and plastic electronic manufacturing due to its low reaction temperatures. This synthetic technique has been employed to prepare $\mathrm{ZnO}$ nanoparticles and other semiconductors successfully. The nanoparticle's crystalline size, morphology, and optical properties can be tuned via the hydrothermal synthesis process by adjusting the precursors' reaction temperature, time, and concentration. ${ }^{1}$ In the present study, the effect of hydrothermally synthesised $\mathrm{ZnO}$ NPs as seeds at various temperature conditions on the $\mathrm{ZnO}$ nanoripples' structural, morphological, and optical properties was investigated and presented.

\section{Experimental}

\subsection{Synthesis of ZnO NPs, unseeded ZnO NRs, and seeded ZnO NRs}

All reagents were analytically pure and used without further purification. To synthesise $\mathrm{ZnO} \mathrm{NPs}$, a stock solution of $2 \mathrm{M}$ zinc acetate dihydrate ( $99.99 \%$ purity) was prepared in $50 \mathrm{ml}$ methanol while stirring. To the stirring stock solution, $0.5 \mathrm{M}$ sodium hydroxide solution prepared in $15 \mathrm{ml}$ methanol was added. The solution was left to go for $30 \mathrm{~min}$. As the solution reached a $\mathrm{pH}$ of $\sim 11$, it was removed and transferred to a Teflon-lined sealed stainless steel autoclave at $140^{\circ} \mathrm{C}$ and $180^{\circ} \mathrm{C}$. Post reaction completion, the resultant white solids were removed. The solids were washed with ethanol, deionised water, and then centrifuged at 
$12000 \mathrm{rpm}$ for $10 \mathrm{~min}$. The washing steps were repeated ten times to remove impurities. The washed products were left to dry at $60^{\circ} \mathrm{C}$ overnight in the oven and packaged in vials.

To produce $\mathrm{ZnO}$-based nanoripples, $0.75 \mathrm{M}$ zinc acetate dihydrate was dissolved in $63 \mathrm{ml}$ of 2-methoxyethanol, and $2.62 \mathrm{ml}$ of ethanolamine was added to stabilise the $\mathrm{Zn}$ (II) ions by facilitating $\mathrm{Zn}$ (II) chelation. The solution was left to stir at $60^{\circ} \mathrm{C}$ for $30 \mathrm{~min}$. Afterward, the solution was divided, and from the two batches of $\mathrm{ZnO}$ NPs, $0.25 \mathrm{mg}$ of $\mathrm{ZnO}$ NPs was drawn from each sample and dissolved in $21 \mathrm{ml}$ of the zinc acetate solution. Two vials were prepared, containing a combination of $\mathrm{ZnO}$ NPs synthesised at $140^{\circ} \mathrm{C}$ and $180^{\circ} \mathrm{C}$ and zinc acetate solution. In preparation for the thin film deposition, the clear quartz substrates were immersed in IPA, acetone, isopropanol, ethanol, and DI water at $60^{\circ} \mathrm{C}$ and sonicated for 15 min each. Compressed nitrogen gas was used to dry the samples after sonication. The thin films were prepared by spin coating the $0.40 \mu \mathrm{L}$ of each solution at $2000 \mathrm{rpm}$ for $40 \mathrm{~s}$ using an Ossila spin coater and ramp-annealed from room temperature to $275^{\circ} \mathrm{C}$ using a Chemat (Technology Inc) hotplate.

\subsection{Characterisation}

A Bruker D8-advanced-8-X-ray powder diffractometer with $\mathrm{CuK}_{\alpha}$ radiation $(\lambda=1.54050 \AA$ ) was used to study the crystallinity and crystal phase of $\mathrm{ZnO}$ NPs. The Bragg angle ranges from $25^{\circ}$ to $85^{\circ}$ with a typical step size of $0.034^{\circ}$. An FEI Tecnai G2 20 field-emission gun (FEG) High-resolution transmission electron microscope (HRTEM) was employed to study the crystallinity, size, and morphological properties of $\mathrm{ZnO}$ NPs.

A Zeiss Auriga emission gun SEM (FEG-SEM) was employed to study the morphological properties of the $\mathrm{ZnO}$-based nanoripples thin films. The electrons' accelerating voltage was set to $5 \mathrm{kV}$ during operation, and secondary electron images were collected with an in-lens detector. Transmittance spectroscopy was utilised to study the optical properties of the ZnO-based nanoripples thin films. Measurements were collected with a Semiconsoft UV-vis spectrometer over a spectral range of $200 \mathrm{~nm}$ to $900 \mathrm{~nm}$.

\section{Results and discussion}

Fig 1 shows the $\mathrm{X}$-ray diffraction patterns of the $\mathrm{ZnO}$ nanopowders produced at $140^{\circ} \mathrm{C}$ and $180^{\circ} \mathrm{C}$, respectively. XRD technique helps to determine the crystal size, sample purity, lattice parameters, and strain due to lattice dislocation. The XRD peaks indicate the high purity of the synthesised ZnO NPs as no foreign phases are detected. These XRD patterns have been indexed as the hexagonal wurtzite phase of $\mathrm{ZnO}^{8}$ with reported lattice constants $a=b=3.24$ $\AA$ and $c=5.21 \AA$ (JPCDS card number: 00-036-1451) ${ }^{8}$. XRD plots of intensity versus $2 \theta$ show sharp peaks (Fig 1) corresponding to particles having crystalline sizes of 21.5 and $21.6 \mathrm{~nm}$ corresponding to $\mathrm{ZnO}$ NPs synthesised at $140^{\circ} \mathrm{C}$ and $180^{\circ} \mathrm{C}$, respectively. ${ }^{9}$ The intensity of the (101) plane is found to be relatively intense for all the samples. Therefore, the preferred orientation is along the [101] axis. $^{10}$

The interplanar distance was calculated according to Bragg's law (3.1), and the crystallite sizes of the maximally intense (101) peaks were estimated using the Debye-Scherrer's formula (3.2). ${ }^{9}$ The lattice strain due to crystal defect and deformation is evaluated using equation (3.3): ${ }^{11}$

$$
\begin{aligned}
& d_{h k l}=n \lambda \sin \theta, \\
& D=\frac{k \lambda}{\beta_{h k l} \cos \theta}, \\
& \varepsilon=\frac{\beta_{h k l}}{4 \tan \theta},
\end{aligned}
$$

here $\mathrm{D}$ is the crystallite size, 0.89 is the Scherrer's constant $k$ for spherical particles, $\lambda$ is the wavelength of the type of incident radiation, $\theta$ is the Bragg diffraction angle, and $\beta$ is the full width at half-maximum (FWHM) of the diffraction peak (101), ${ }^{7}$ and $\varepsilon$ is the lattice strain. The lattice parameters were calculated from equation (3.3),

$\frac{1}{d_{h k l}^{2}}=\frac{4}{3\left[a^{2}\left(h^{2}+h k+k^{2}\right)\right]}+\frac{l^{2}}{c^{2}}$

where $d_{h k l}$ is the interplanar spacing for the plane (101), $a$ and $c$ are the lattice parameters, and $h, k$, and $l$ are the Miller indices (7). ${ }^{7}$ The (101) plane was used to make a quantitative analysis presented in Table 1. Table 1 shows the estimated crystallite sizes, lattice parameters, and lattice strain of $\mathrm{ZnO}$ NPs fabricated at $140^{\circ} \mathrm{C}$ and $180^{\circ} \mathrm{C}$, respectively. Therefore, the lattice dislocations found in the sample are associated with the strain calculated and the change in the crystallite size. This is supported by the deviation in the lattice parameters values from the reported values, ${ }^{8}$ outlined in Table 1. However, the c/a ratios shown in Table 1 for the two $\mathrm{ZnO}$ NPs samples are 1.61. This axial ratio value confirms that the atoms of the two ZnO NPs samples are closely packed, just as in an ideal $\mathrm{ZnO}$ hexagonal close-packed crystal value at $1.60{ }^{7}$

HRTEM images of hydrothermally grown $\mathrm{ZnO}$ samples are shown in Fig 2 (a) and (b). The average particle sizes were calculated; the histograms alongside the SAED micrographs are presented in Fig 2 (a) and (b) inset. A combination of primarily spherical nanostructures and a small yield of nanorods is observed in Fig 2 (a). An increase in temperature to $180^{\circ} \mathrm{C}$ (Fig 2 (b)) resulted in a combination of primarily nanorods and secondarily sphere-

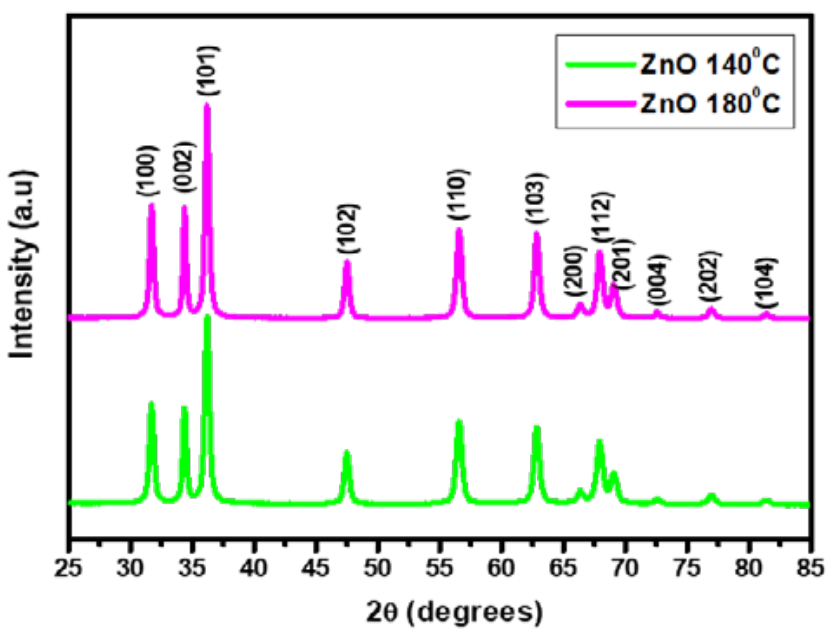

Figure 1: (a) XRD pattern of $\mathrm{ZnO}$ NPs synthesized $140^{\circ} \mathrm{C}$ and $180^{\circ} \mathrm{C}$, respectively 
Table 1: A summary of the estimated lattice parameters, calculated crystallite size, and microstrain for $\mathrm{ZnO}$ synthesised $140^{\circ} \mathrm{C}$ and $180^{\circ} \mathrm{C}$, respectively

\begin{tabular}{lccccc}
\hline \multirow{2}{*}{ Sample } & \multicolumn{3}{c}{ Lattice parameters $(\mathbf{A})$} & (Crystallite size) & \multirow{2}{*}{ Lattice Strain } \\
\cline { 2 - 5 } & $\mathbf{a}$ & $\mathbf{c}$ & $\mathbf{c} / \mathbf{a}$ & $\mathbf{D}(\mathbf{n m})$ & $5.90 \times 10^{-4}$ \\
\hline $\mathrm{ZnO} N$ Ps $\left(140^{\circ} \mathrm{C}\right)$ & 3.25 & 5.23 & 1.61 & 21.5 & $5.95 \times 10^{-4}$ \\
ZnO NPs $\left(180^{\circ} \mathrm{C}\right)$ & 3.23 & 5.21 & 1.61 & 21.6 & 5 \\
\hline
\end{tabular}
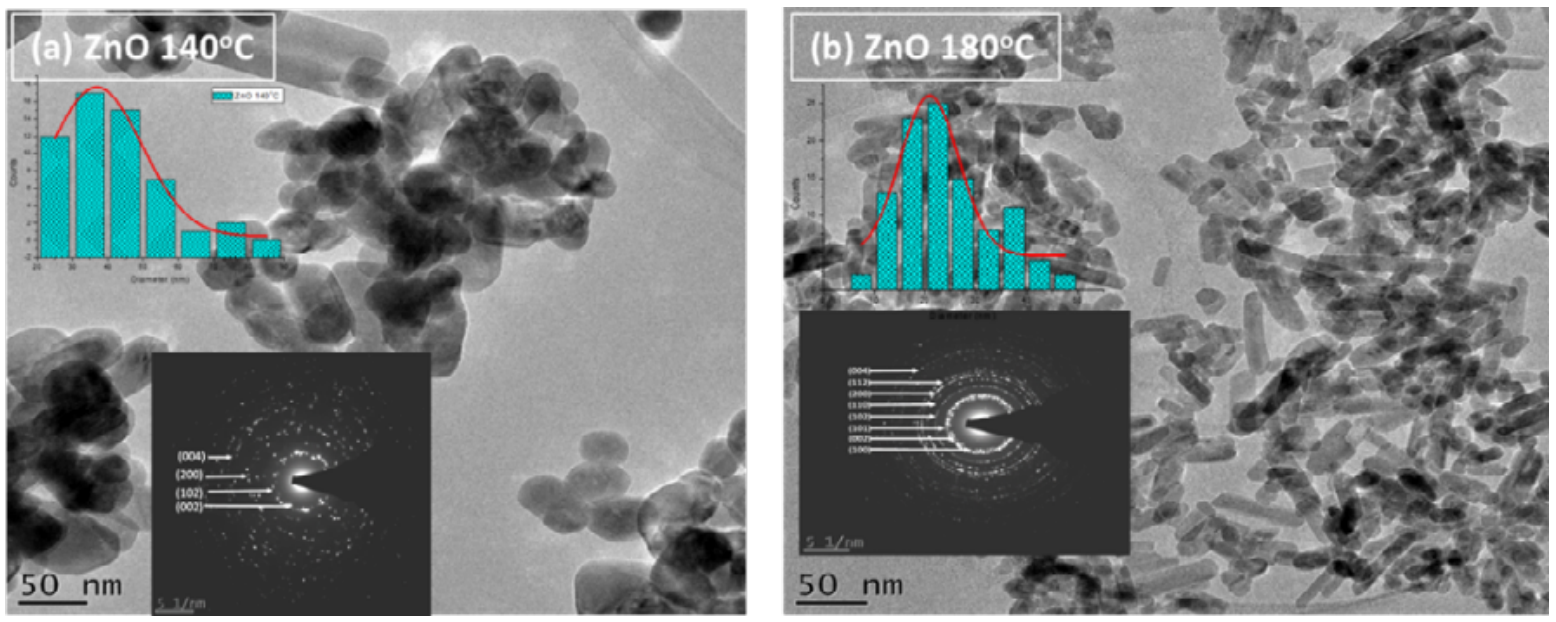

Figure 2: TEM images of $\mathrm{ZnO}$ NPs fabricated at $140^{\circ} \mathrm{C}$ and $180^{\circ} \mathrm{C}$. The inset show diameter distributions and SAED images
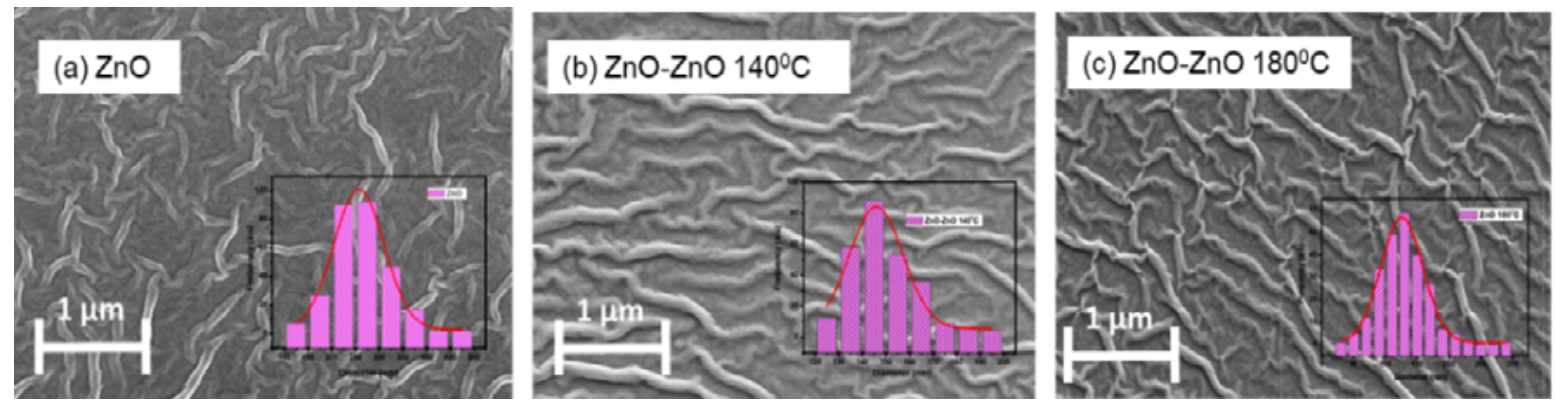

Figure 3: SEM images of (a) unseeded ZnO NRs, (b) and (c) seeded ZnO NPs-ZnO NRs, synthesised with bare zinc acetate solution combined with $\mathrm{ZnO}$ NPs synthesised at $140^{\circ} \mathrm{C}$ and $180^{\circ} \mathrm{C}$, respectively. The inset images show the diameter distribution of the nanoripples

shaped $\mathrm{ZnO}$ NPs. The particle sizes of these samples decrease from $36.9 \mathrm{~nm}$ to $15.7 \mathrm{~nm}$ with increasing temperature. The inset of the histograms demonstrates this in Fig 2 (a) and (b), and it is consistent with the reported $\mathrm{ZnO}$ structure of Sonima Mohan et al. ${ }^{10}$ The observed diffraction spots and ring patterns in SAED micrographs were indexed with the assistance of bulk $\mathrm{ZnO}$ JCPDS card 00-036-1451 data. ${ }^{8}$ The SAED patterns illustrate that the samples are hexagonal wurtzite $\mathrm{ZnO}$ structures in crystallography, which agrees with XRD results. Their particle size determined from TEM analysis of $\mathrm{ZnO}$ NPs at $140^{\circ} \mathrm{C}$ is greater than the Scherrer method-extracted crystallite size in XRD analysis. Furthermore, the particle size of $\mathrm{ZnO}$ NPs at $180^{\circ} \mathrm{C}$ was found to be less than the crystallite size. The intensity of the diffraction rings increases with the synthesis temperature of the ZnO NPs, which suggests an increase in the polycrystallinity of the nanomaterials. ${ }^{7}$

The morphology and diameter distribution of the unseeded $\mathrm{ZnO}$ NRs and seeded $\mathrm{ZnO}$ NRs fabricated from a combination of hydrothermally synthesised $\mathrm{ZnO}$ nanoparticles at $140^{\circ} \mathrm{C}$ and $180^{\circ} \mathrm{C}$, and bare zinc acetate solution NRs are presented in the
SEM images in Fig 3 (a)-(c). Seedless $\mathrm{ZnO}$ nanoripples show contracted nanoripple structures with rugged ends. SEM images illustrated that seeding the $\mathrm{ZnO}$ nanoripples causes the nanoripples to elongate and uniformly form on the substrate. Furthermore, an increase in the temperature of the seeding nanoparticles causes a decrease in the diameter distribution of the nanoripples (Fig 3 (a) and (b) inset). The diameter distribution of unseeded $\mathrm{ZnO}$ NRs is $320.4 \pm 86.0 \mathrm{~nm}, \mathrm{ZnO}-\mathrm{ZnO} 140^{\circ} \mathrm{C}$ NRs is $246.4 \pm 74.7 \mathrm{~nm}$, and $\mathrm{ZnO}-$ $\mathrm{ZnO} 180^{\circ} \mathrm{C}$ NRs $125.8 \pm 33.0 \mathrm{~nm}$. Seeding the nanoripples causes the thin film to decrease in thickness. The thickness values of the thin films were found to be 188,100 , and $96 \mathrm{~nm}$ for unseeded $\mathrm{ZnO}$ $\mathrm{NRs}, \mathrm{ZnO}-\mathrm{ZnO} 140^{\circ} \mathrm{C}$, and $\mathrm{ZnO}-\mathrm{ZnO}$ NRs $180^{\circ} \mathrm{C}$, respectively. The decrease in the thickness attributes to the transparency in thin films. An increase in transparency is beneficial for optoelectronic applications as absorption of charge carriers increases due to high transparency. ${ }^{12}$

The UV-Visible absorbance spectra, transmittance spectra, and Tauc plots of the unseeded $\mathrm{ZnO}$ nanoripple thin film structures and seeded $\mathrm{ZnO}-\mathrm{ZnO} 140^{\circ} \mathrm{C}$ NRs, and $\mathrm{ZnO}-\mathrm{ZnO} 180^{\circ} \mathrm{C}$ NRs 

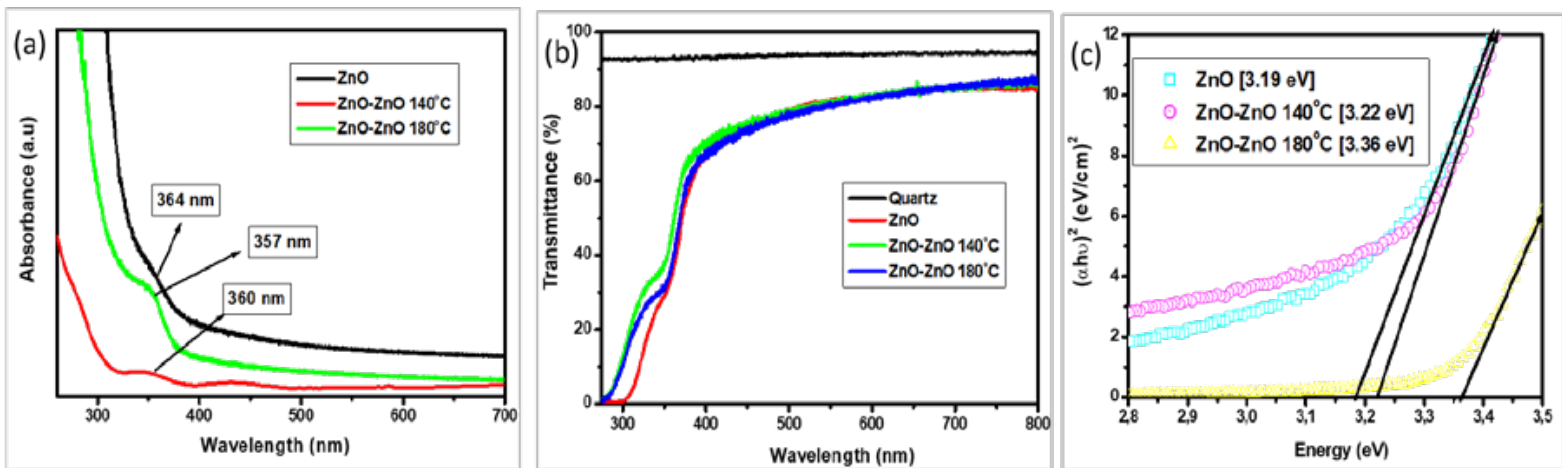

Figure 4: (a) the absorbance spectra (b), UV-Vis transmittance plots spectra, and (c) the Tauc plots representing direct band gap values the unseeded $\mathrm{ZnO}$ NRs and seeded $\mathrm{ZnO}$ NPs-ZnO NRs synthesised with bare zinc acetate solution combined with ZnO NPs synthesised at $140^{\circ} \mathrm{C}$ and $180^{\circ} \mathrm{C}$, respectively

synthesised on quartz substrates are shown in Fig 4 (a), (b), and (c) respectively.

The absorption spectra (Fig 4 (a)) show near UV absorption at $\sim 357, \sim 364$, and $\sim 360 \mathrm{~nm}$ for $\mathrm{ZnO} \mathrm{NRs}, \mathrm{ZnO}-\mathrm{ZnO} 140^{\circ} \mathrm{C} \mathrm{NRs}$, and $\mathrm{ZnO}-\mathrm{ZnO} 180^{\circ} \mathrm{C}$ NRs, respectively. This change is a blue-shift compared to the bulk value $(\sim 375 \mathrm{~nm})^{10}$ and is attributed to the intrinsic defects. All the samples fabricated show transmission of $\mathrm{T}>75 \%$ at the longer wavelength of $\lambda>550 \mathrm{~nm}$ in the visible region of the electromagnetic spectrum (Fig 4 (b)). The transparency is attributed to the morphology alteration of the NRs due to the incorporation of $\mathrm{ZnO}$ nanoparticles. Band gap values for the three samples were determined from Tauc plots. The values were extrapolated from the linear portion of the $\mathrm{x}$-axis of $(\alpha h v)^{1 / 2}$ versus $h v$ plot as $(\alpha h v)^{1 / 2}$ approaches zero, shown in Fig 4 (c). The obtained values are 3.19, 3.22, and $3.36 \mathrm{eV}$ for unseeded $\mathrm{ZnO}$ NRs, $\mathrm{ZnO}-\mathrm{ZnO} 140^{\circ} \mathrm{C} \mathrm{NRs}$, and $\mathrm{ZnO}-\mathrm{ZnO} 180^{\circ} \mathrm{C}$ NRs respectively. In optoelectronics, large band gap value(s) of $\mathrm{ZnO}$ nanostructured thin films ranging from 3.0-3.4 eV are essential for effective working devices. ${ }^{13}$ For example, Yong-Jin Noh et al. ${ }^{14}$ optimised $\mathrm{ZnO}$ nanostructures for Inverted polymer solar cells application. The optimised $\mathrm{ZnO}$ layer exhibit a band gap of $\sim 3.2 \mathrm{eV}$ and an efficiency of $4.22 \%$. Therefore, the optimised $\mathrm{ZnO}$ layer in inverted organic cells is recognised as a potential material for the electron transport layer to substitute $\mathrm{TiO}_{2}$. Thus, the band gap values found in this study ranging from 3.19 to $3.36 \mathrm{eV}$ agree with the intrinsic band gap of $3.37 \mathrm{eV} .{ }^{10}$ The increasing band gap with increased NPs' synthesis temperature leads to a change in the Plasmon absorption region that is attributed to the $\mathrm{ZnO}$ NPs aggregation. ${ }^{7}$

\section{Conclusion}

$\mathrm{ZnO}$ NPs were fabricated by the hydrothermal method under various reaction temperature values to study the effect of the synthesised $\mathrm{ZnO}$ NPs as seeds on the properties of $\mathrm{ZnO}$ NRs. In the analysis, TEM images revealed that hydrothermal synthesis of $\mathrm{ZnO}$ NPs at different temperature values results in NPs of various shapes. The particle size values were found to be less than the crystallite sizes obtained in XRD. The unseeded ZnO NRs thin films showed a diameter distribution of $320.4 \pm 86.0 \mathrm{~nm}$, which decreased as the temperature of the ZnO NPs seeds increased. Adding the $\mathrm{ZnO}$ NPs produced at $140^{\circ} \mathrm{C}$ and $180^{\circ} \mathrm{C}$ leads to a decrease in the diameter distributed along the area. All the thin films were fabricated with $0.40 \mu \mathrm{L}$, yet each sample resulted in different thicknesses, illustrating that the thin films are highly affected by adding the ZnO NPs seeds. Therefore, a change in the
UV/Vis absorption occurred, and the seeded samples showed a blue shift compared to the unseeded sample, with band gap values ranging from $\sim 3.19-3.36 \mathrm{eV}$. This analysis suggests that adding $\mathrm{ZnO}$ NPs in the fabrication of $\mathrm{ZnO}$ NRs improves the properties of $\mathrm{ZnO}$ NRs thin films. Therefore, $\mathrm{ZnO}$ NPs-ZnO NRs may be used as a potential material for optoelectronic applications.

\section{Acknowledgments}

This work was financially supported by the Nanoscience Postgraduate Programme, South Africa. The authors would like to acknowledge iThemba labs, Faure, Cape Town, the CSIR group, The University of the Western Cape Microscope Unit, and Siyabonga Mdluli at UWC Chemical Sciences measurement assistance.

\section{References}

1. Aneesh PM, Vanaja KA, Jayaraj MK. 2007. Synthesis of ZnO nanoparticles by hydrothermal method, Optoelectronic Devices Laboratory, Vol. 6639, pp. 1-9.

2. Rodnyi PA, Khodyuk IV. 2011. Optical and Luminescence Properties of Zinc Oxide (Review). 5, Condensed matter, Vol. 11, pp. 814-824.

3. Nagayasamy N, Gandhimathination S, Veerasamy V. 2013. The Effect of ZnO Thin Film and Its Structural and Optical Properties Prepared by Sol-Gel Spin Coating Method. Open Journal of Metal, Vol. 3, pp. 8-11.

4. Janardhan E, Reddy MM, Reddy PV, Reddy MJ. 2017. Synthesis of $\mathrm{ZnO}$ Nanoparticles - A Hydrothermal Protocol. 6, European Journal of Applied Sciences, Vol. 9, pp. 312-314.

5. Muhammad A, Ili RR, Faisal G, Shams U-R, Faheem BK, Rafaqat H. 2018. Continuous facile synthesis of nano-sized zinc oxide and its optical properties. 7, Materials Research Express, Vol. 5.

6. Huang J, Yin Z, Zheng Q. 2011. Applications of $\mathrm{ZnO}$ in organic and hybrid solar cells. Applied Physics, Vol. 4, pp. 3861-3877.

7. Soni BH, Deshpande MP, Bhatt SV, Garg N, Chaki SH. 2013. Studies on $\mathrm{ZnO}$ Nanorods Synthesized by Hydrothermal Method and their Characterization. 4, Journal of nano- and electronic Physics, Vol. 5, pp. 04077(1-6).

8. Chung FH. 1974. Journal of Applied Crystallography. Vol. 7, p. 519.

9. Cullity BD. 1978. Elements of X-ray diffraction. s.l. : Addison-Wesley Pub. Co, p. 555.

10. Mohan S, Vellakkat M, Aravind A, Reka U. 2020. Hydrothermal synthesis and characterization of Zinc Oxide nanoparticles of various shapes under different reaction conditions, Nano Express, Vol. 030028 , pp. $1-15$.

11. Suryanarayana C, Norton MG. 1998. Determination of Crystallite Size and Lattice Strain. X-Ray Diffraction. New York: Springer Science+Business Media, pp. 207-221.

12. Muhammad BL, Cummings F. 2019. Nitrogen plasma treatment of $\mathrm{ZnO}$ and $\mathrm{TiO} 2$ nanowire arrays for polymer photovoltaic applications, Surfaces, and Interfaces, Vol. 17, p. 100382.

13. Alias SS, Ismail AB, and Mohamad AA. 2010. Effect of $\mathrm{pH}$ on $\mathrm{ZnO}$ nanoparticle properties synthesized by sol-gel centrifugation. 2, Journal of Alloys and Compounds, Vol. 499, pp. 231-237.

14. Noh Y-J, Na S-I, Kim S-S. 2013. Inverted polymer solar cells including $\mathrm{ZnO}$ electron transport layer fabricated by facile spray pyrolysis, Solar energy materials, and solar cells, Vol. 117, pp 139-144. 